This document was prepared in conjunction with work accomplished under Contract No. DE-AC09-96SR18500 with the U.S. Department of Energy.

This work was prepared under an agreement with and funded by the U.S. Government. Neither the U. S. Government or its employees, nor any of its contractors, subcontractors or their employees, makes any express or implied: 1 . warranty or assumes any legal liability for the accuracy, completeness, or for the use or results of such use of any information, product, or process disclosed; or 2 . representation that such use or results of such use would not infringe privately owned rights; or 3 . endorsement or recommendation of any specifically identified commercial product, process, or service. Any views and opinions of authors expressed in this work do not necessarily state or reflect those of the United States Government, or its contractors, or subcontractors. 


\title{
Uranium Detection Using Small Scintillators in a Maritime Environment
}

\author{
K. J. Hofstetter, D. M. Beals, D. M. Odell \\ Savannah River National Laboratory \\ Aiken, SC 29808
}

\begin{abstract}
The performance of several commercially available portable radiation spectrometers containing small $\mathrm{NaI}(\mathrm{Tl})$ scintillation detectors has been studied at the Savannah River National Laboratory (SRNL). These hand-held radioisotope identifiers are used by field personnel to detect and identify the illegal transport of uranium as a deterrent to undeclared nuclear proliferation or nuclear terrorism. The detection of uranium in a variety of chemical forms and isotopic enrichments presents some unique challenges in the maritime environment. This study was conducted using a variety of shielded and unshielded uranium sources in a simulated maritime environment. The results include estimates of the detection sensitivity for various isotopic enrichments and configurations using the manufacturer's spectral analysis firmware. More sophisticated methods for analyzing the spectra off-line are also evaluated to determine the detection limits and enrichment sensitivities from the field measurements.
\end{abstract}

\section{Introduction}

Reliable methods for the detection and identification of uranium is very important since

${ }^{235} \mathrm{U}$ can be used to construct a nuclear explosive device if a sufficient quantity can be assembled in such a way to produce an uncontrolled chain reaction. A nuclear proliferator or a terrorist may attempt to obtain a sufficient quantity of highly enriched uranium to incorporate it into the design of an improvised nuclear device (IND).

Normal uranium has $0.72 \%$ by weight of this fissile ${ }^{235} \mathrm{U}$ isotope. There are a variety of physical or chemical methods to increase the isotopic content of ${ }^{235} \mathrm{U}$ in uranium.

According to the IAEA, low enriched uranium (LEU) includes all enrichments up to $20 \%$

${ }^{235} \mathrm{U}$. Light water reactor fuel is typically enriched in ${ }^{235} \mathrm{U}$ to $\sim 5 \%$. Research reactors and nuclear submarines require moderately high enrichments of uranium as fuel. Weapons grade uranium (WGU) is any enrichment greater than $80 \%$. The IAEA defines a "significant quantity" of WGU at $10 \mathrm{~kg}$. It is generally agreed that $25 \mathrm{~kg}$ of uranium enriched to greater than $90 \%$ is necessary to produce a nuclear explosive. (1)

To detect a diversion of highly enriched uranium (HEU), the radiations being emitted from the source must quickly and efficiently be detected and analyzed by field agents using hand-held radiation detection devices. Uranium-235 emits alpha radiations and relatively low energy gamma radiations which have limited penetration through common materials. Uranium-235 does not emit neutrons from spontaneous fission but can cause neutrons to be emitted from $(\alpha, n)$ reactions on low-Z material in the source. The authors performed a detailed examination of the very low energy gamma radiations using room 
temperature $\mathrm{CdZnTe}$ and CdTe semiconductor detectors for detecting small quantities of enriched uranium and presented the results in reference 2.

Uranium is found in a number of harmless products as naturally occurring radioactive material (NORM). Since natural uranium has several isotopes and can be found in many forms, identifying uranium isotopes of concern is a complex task. In some NORM or commercial sources, all the radioactive decay progeny are present in nuclear equilibrium amounts. In those cases, the gamma radiations are dominated from the decay of the radioisotope ${ }^{226} \mathrm{Ra}$ and its progeny. In other items, the chemical processing to produce the final product removes these long-lived daughters leaving only the immediate uranium progeny which emit their characteristic gamma-ray signatures.

The major gamma ray emitted in the decay of ${ }^{235} \mathrm{U}$ is $185.7 \mathrm{keV}$. A gamma ray of equivalent energy is also emitted in the decay of ${ }^{226} \mathrm{Ra}$, a ubiquitous radioisotope found in NORM and many commercial products. The major source of this isotope $\left({ }^{226} \mathrm{Ra}\right)$ is from the decay of the naturally occurring uranium isotope ${ }^{238} \mathrm{U}$. Other radiations from the decay of the ${ }^{226} \mathrm{Ra}$ progeny, ${ }^{214} \mathrm{Bi}$ and ${ }^{214} \mathrm{~Pb}$, result in a unique gamma signature which can mask the presence of ${ }^{235} \mathrm{U}$. While ${ }^{226} \mathrm{Ra}$ is in the decay chain of naturally occurring uranium, it does not exist in the spectrum of radiations from isotopically enriched uranium.

The gamma rays at 92.4 and $92.8 \mathrm{keV}$ arising from ${ }^{234} \mathrm{Th}$, the decay daughter of ${ }^{238} \mathrm{U}$, and the $185.7 \mathrm{keV}$ line from ${ }^{235} \mathrm{U}$ are most often used to determine the isotopic enrichment of suspicious uranium bearing materials using low resolution scintillation devices. The ratio of the intensity of these gamma rays is affected by internal self absorption and external shielding. A typical spectrum of unshielded HEU is shown in Figure 1 as recorded with a small $\mathrm{NaI}(\mathrm{Tl})$ spectrometer. Note the two peaks at 93 and $186 \mathrm{keV}$. The peak at $144 \mathrm{keV}$ also arises from the decay of ${ }^{235} \mathrm{U}$.

The low energy portion of the spectrum up to $\sim 100 \mathrm{keV}$ is affected by instrumental effects (built-in low energy discriminator to eliminate detector noise) and detector encapsulation materials, the self-absorbance of the radiations in the sample, and the presence of X-rays caused by self fluorescence in the sample. These will be discussed in more detail in a later section of this paper.

The initial detection of suspicious uranium-containing material will probably be made by a person wearing a personal radiation pager or by a fixed radiation portal monitor at a vehicle choke point. The normal terrestrial background dose rate is $10-20 \mu \mathrm{R} / \mathrm{hr}$ as measured by instruments calibrated against a Cs-137 source. In the maritime environment, gamma backgrounds of $1-2 \mu \mathrm{R} / \mathrm{hr}$ are routinely encountered when boarding vessels at sea. Most radiation pagers contain a CsI scintillation detector coupled to a PIN photodiode. When initially activated, they determine the count rate in the area for a brief interval $(\sim 30 \mathrm{sec})$ and the typical error based on simple counting statistics. The alarm level, converted to dose rate, is then computed by the firmware to be a multiple of the standard deviation above the calculated background. To minimize false alarms, this multiple may be $10 \mathrm{X}$ which is deemed adequate in very low background, e.g. maritime, 
environments. The probability of detection is then based on the dose rate of the illicit source since the background contribution is almost negligible.

For example, to test the ability to detect a significant quantity of $\mathrm{HEU}\left(90 \%{ }^{235} \mathrm{U}\right)$ at sea consider the following two examples:

1. A $5 \mathrm{~kg}$ ingot of HEU metal will generate a radiation dose rate field of $\sim 6 \mu \mathrm{R} / \mathrm{hr}$ at 2 meters from a detector, the approximate center of a cargo container. If the container is filled with bulk material with average density of $0.5 \mathrm{gm} / \mathrm{cm}^{3}$, the dose rate drops to a few $\mathrm{nR} / \mathrm{hr}$. If the $5 \mathrm{~kg}$ sample is low-fired oxide, the dose rate increases to $\sim 30 \mu \mathrm{R} / \mathrm{hr}$ at 2 meters with no other materials in the container. These calculations were performed using the standard dose rate simulation code MicroShield $-4^{\mathrm{TM}}$.

2. A $40 \mathrm{gm}$ sample of HEU inside a 0.25 inch metal can is calculated to generate a $\sim 200 \mu \mathrm{R} / \mathrm{hr}$ dose rate at $18 \mathrm{~cm}$. While the 40 gm sample could possibly be detected on land, the $5 \mathrm{~kg}$ sample would probably not trigger a terrestrial alarm at 2 meters.

To resolve unanticipated alarms, a small hand-held RIID with spectroscopic capability is necessary to determine the radioisotopes responsible for the increased radiation. If the RIID identifies ${ }^{235} \mathrm{U}$ as a possible radiation source based on a spectrum similar to that shown above (Figure 1), a suitable follow-on response is initiated.

Most current commercial off-the-shelf (COTS) hand-held radioisotope identifiers (RIID) contain a small $\mathrm{NaI}(\mathrm{Tl})$ scintillator with built-in multichannel analyzer and custom firmware to identify the isotopes responsible for the increased gamma radiation field. These detectors are intended to discriminate between nuclear threat materials and commonly encountered legitimate gamma-ray sources. The detection and identification of the isotope ${ }^{235} \mathrm{U}$ at levels sufficient to present reasonable cause for continued investigations is necessary for continued national security. A suite of these COTS instruments were tested at SRNL in a simulated maritime environment. The performance of selected instruments has also been field tested in an extended maritime background study on board ships at a number of maritime ports of entries $(\underline{\mathbf{3}})$. The results of the study reported here represent a systematic evaluation of these RIIDs when exposed to ${ }^{235} \mathrm{U}$ sources as compared to measurements using a portable high resolution high purity germanium (HPGe) spectrometer.

\section{Instrumentation}

Four different RIIDs were tested in this study which represent those commonly used by US Customs and Border Protection, federal and local law enforcement agents, Port Security officials and the US Coast Guard. Trained field agents use this equipment to resolve radiation alarms received by personal radiation pagers or radiation portal monitors (RPM). The hand-held RIIDs provide the field agents with an initial identification of the possible source of radiation, i.e, medical, NORM, commercial or 
nuclear. In addition to identifying the category of the radiation, they also identify the possible radioisotopes present. In this study these RIID instruments were used to record gamm-ray spectra of a variety of uranium sources and samples that have been carefully characterized for chemical and isotopic content. The experiments were performed in a low background shield made from pre-world war II battleship iron armor plate $(30 \mathrm{~cm})$ with a thin $\mathrm{Pb}$ line; the background inside this shield is $1-2 \mu \mathrm{R} / \mathrm{hr}$, similar to that found on a ship at sea. The sources were positioned at known distances from the detector face and the spectra were recorded using the manufacturer's recommended counting interval (typically 30-60 seconds). A brief description of each RIID used in this study is given below.

The IdentiFINDER-U is a handheld isotopic identification device manufactured by Thermo Electron Corporation. The IdentiFINDER-U is an underwater version of the FieldSpec and contains a $(31 \mathrm{~mm} \phi$ x $38 \mathrm{~mm}) \mathrm{NaI}(\mathrm{Tl})$ scintillation crystal for detecting gamma radiation, a He-3 detector for neutron indication, and a GM tube for measuring high dose rates. The instrument provides nuclide identification, dose rates and total dose display, and can be used in search mode for locating sources. For identification purposes, there are 72 reference spectra in the nuclide firmware library which are divided into the four main categories. The user can measure and add up to 10 additional reference spectra in the firmware library. The instrument stores dose rate, spectral, and isotopic information which can be downloaded to a PC using an RS232 serial output. The standard spectrum length is 1024 channels $(50-1500 \mathrm{keV})$ and 50 spectra can be stored in the memory. The instrument has an internal Cs-137 source $(15 \mathrm{nCi})$ which permits automatic energy and temperature stabilization, detector efficiency, and automatic dose calibration. Quantitative off-line analysis software (winTMCA ${ }^{\mathrm{TM}}$ ) is available from the manufacturer.

Two SAM-935's were used in this study, one with an internal $\mathrm{NaI}(\mathrm{Tl})$ (38 $\mathrm{mm} \phi$ x 50 $\mathrm{mm})$ detector and one with an external $51 \mathrm{~mm} \phi$ x $51 \mathrm{~mm} \mathrm{NaI(Tl)} \mathrm{detector.} \mathrm{The} \mathrm{SAM-}$ 935 is a portable surveillance and monitoring system which also makes nuclide identifications. It is manufactured by Berkeley Nucleonics Corporation. The instrument can be used in the real time or manual multichannel analysis mode. Real time functions include dose rate, spectrum analysis mode, and monitor mode. The dose rate mode allows the operator to use one of four buttons to perform calibrations, search, and record spectra from the same display screen while the monitor mode shows a histogram with isotope intensity as a function of standard deviations above background. The spectrum analysis mode displays a graphic spectrum reflecting any peaks (nuclides) detected. The MCA mode is menu driven. Isotope identification is performed in real time as the spectrum is accumulated. A moderated He-3 proportional neutron detector is included. The SAM-935 must be calibrated with external sources prior to use. The energy calibration must be performed any time a large temperature change occurs. The preset energy range for the unit is $18-3000 \mathrm{keV}$. The SAM-935 is capable of storing 250 spectra in an energy compressed proprietary format. Downloading of the data is possible using a standard RS-232 serial port. Quantitative off-line analysis software (QtmMCA ${ }^{\mathrm{TM}}$ ) is available from the manufacturer. 
The GR-135 is manufactured by Exploranium Radiation Detection Systems and contains a $38 \mathrm{~mm} \phi$ x $55 \mathrm{~mm} \mathrm{NaI}(\mathrm{Tl})$ detector. The hand-held instrument is intended for use both as a search tool and an identification tool. It operates in dose rate (or search) mode as well as ID mode. Either of these modes can be accessed through automatic or manual operation of a one button joystick which makes one hand operation possible. The GR-135 is placed in a docking station which has a charger for the batteries and a $0.25 \mu \mathrm{Ci} \mathrm{Cs}-137$ calibration source. The gain is thermally compensated using a built-in thermistor when undergoing large temperature variations. The GR-135 is capable of storing 40,000 dose measurements or 2501024 channel spectra. The stored data is downloaded upon replacing the instrument into the docking station when connected to a PC through the RS232 serial port. The IdentiView ${ }^{\mathrm{TM}}$ software, available from the manufacturer, is necessary for data downloads. Neutrons are detected using a solid state Lithium glass detector. A Geiger Mueller detector is available for higher dose rate measurements. Detection capabilities include radionuclides with gamma emissions in the $20-3000 \mathrm{keV}$ range. Five nuclide libraries are available in the firmware for nuclide identification; four of which are preset and one that can be user defined.

\section{Experimental}

Small samples (1-50 gm) of uranium of known enrichments were positioned near the front face of the RIIDs at the most sensitive measurement location and spectra were recorded for a short counting interval (30-60 seconds). Positioning of the samples was based on the measured dose rate and maintained $<200 \mu \mathrm{r} / \mathrm{hr}$. All experiments were performed in the low background shield (dose rate $<1 \mu \mathrm{R} / \mathrm{hr}$ ). Figure 2 shows the low energy spectra recorded with each instrument of an enriched $\left(68 \%{ }^{235} \mathrm{U}\right)$ uranium source. The spectra were analyzed using the manufacturer's embedded firmware and the spectra stored in the internal memory. After the data acquisition campaign was completed, the spectra were downloaded to a personal computer for off-line analyses. Except for a few instances, the uranium samples had been separated from their equilibrium decay progeny such that the radiations from ${ }^{238} \mathrm{U}$ could be seen in the samples. In general, the RIIDs were able to identify the presence of enriched uranium at enrichments above $5 \%$ by weight. Below that value, the detection of LEU, natural and DU was sporadic. At low enrichments, the RIIDs frequently reported the presence of ${ }^{238} \mathrm{U}$ in the spectra. No estimate of the quantity of enriched material is made by the RIIDs. For those estimates, the spectra and counting conditions are sent to a subject matter expert for detailed analyses.

For the samples examined in this study, the manufacturer's PC-based software was able to extend the detection level of ${ }^{235} \mathrm{U}$ to a lower enrichment through the subtraction of backgrounds and more sophisticated analyses of the peaks in the spectra. For example, a 30 second spectrum of a sample with $1.21 \%$ enrichment taken with an IdentiFINDER-U was identified as containing ${ }^{235} \mathrm{U}$ and ${ }^{238} \mathrm{U}$ at a $50 \%$ confidence level using the winTMCA ${ }^{\mathrm{TM}}$ software but was unable to make the detection using the hand-held instrument firmware. In a depleted uranium sample $(<0.2 \%$ enrichment $)$, the winTMCA ${ }^{\mathrm{TM}}$ software identified ${ }^{238} \mathrm{U}$ at the $70 \%$ confidence level with only a trace of ${ }^{235} \mathrm{U}(<10 \%$ confidence). 


\section{Results and Discussion}

To estimate the isotopic content of a suspect uranium sample, the spectra obtained with the RIIDs must be analyzed using advanced spectroscopic techniques. An understanding of the inherent resolution and efficiency limitations of small scintillators and the local environment is required. Since the spectra are affected by external shielding and many intrinsic parameters (mass, chemical form, density, etc.), the analysis methods must accommodate these variables in a formal manner. One method of analysis is multiple linear regression of full energy standard spectra combining absorption, scattering and other interferences for an overall best fit. This technique was developed by Mitchell et.al (4) and has been used to analyze the spectra recorded in this study. As an example of this method, a 180-second spectrum of a fiestaware plate was recorded with an IdentiFINDER-U spectrometer and downloaded to a PC for analysis. The GADRASw computer code was used to analyze the resulting spectrum. The results of this analysis uncorrected for background are shown in Figure 3.

The ${ }^{137} \mathrm{Cs}$ component in the spectrum is due to the internal source in the instrument. The results of this analysis indicated the isotopic content of the uranium in this sample is $0.9 \pm 0.2 \%{ }^{235} \mathrm{U}$ confirming its natural abundance. Most of the hand-held instruments indicated the fiestaware contained enriched uranium due to the templates in memory used for identification. A general tendency to overestimate the ${ }^{235} \mathrm{U}$ content in samples and artifacts with low enrichments was observed for the RIIDs tested. By performing a similar detailed analysis of the spectra generated in this study, some general conclusions concerning the reason for this performance resulted.

As an example, consider the spectrum of radiations observed from a 1 gram sample of a NIST-traceable standard containing $10 \%{ }^{235} \mathrm{U}$ taken for 60 seconds in the low background shield. The resulting spectrum taken at $5 \mathrm{~cm}$ from the source is shown in Figure 4. The GADRASw analysis was performed with only the ${ }^{235} \mathrm{U},{ }^{238} \mathrm{U}$ and ${ }^{137} \mathrm{Cs}$ components selected for inclusion in the regression analysis. The low energy portion of the spectrum has been expanded to show more detail. The results indicate an approximate enrichment of $2 \pm 1 \%{ }^{235} \mathrm{U}$.

The results of the GADRAS analysis clearly show an unidentified component in the spectrum around $100 \mathrm{keV}$. Since the sample was a pure uranium oxide sample, there is sufficient alpha activity in the sample to cause it to self-fluoresce and generate uranium $\mathrm{X}$-rays from the ionization cause by the internal alpha radiation. If an unknown uranium $\mathrm{X}$-ray component is added to the spectrum, the fit is markedly improved as shown in the following figure (Figure 5). The enrichment is determined to be $8.5 \pm 0.8 \%{ }^{235} \mathrm{U}$ following this analysis. Additional improvements could be made by modeling the source to include self absorption and scattering effects.

To test this phenomenon, a number of spectra of the samples used in this study were taken with a high resolution gamma-ray spectrometer. Gamma-ray signatures can be obtained through measurements using high-purity germanium (HPGe) detectors on well 
characterized samples. These high resolution spectrometers provide unambiguous identification of nuclear weapons materials with high precision but are rarely available to field inspection personnel. A typical spectrum of an enriched sample taken with a HPGe spectrometer and analyzed by the GADRASw code, is shown in Figure 5. The suite of radioisotopes selected for inclusion in the multi-regression analysis is the same for both Figure $6 \mathrm{~A}$ and $6 \mathrm{~B}$, however Figure 5B includes the uranium $\mathrm{X}$-rays from selffluorescence. Note the improvement in the Chi-square fit to the data in Figure 6B. The resulting isotopic enrichment for each case was calculated to be $40 \%$ and $61 \%$ respectively. In both cases, the intensity of the $186 \mathrm{keV}$ gamma-ray from ${ }^{235} \mathrm{U}$ is slightly underestimated and suggests self-absorption has not been adequately considered in the calculation.

Since the alpha specific activity of enriched uranium samples increases with enrichment, the fraction of X-rays produced by self-fluorescence should increase proportionately. This hypothesis was tested using the results of several spectra taken with the HPGe detector and analyzed by the GADRASw code. These results are summarized in Table 1. The results indicate that including the $\mathrm{X}$-ray component in the suite of radionuclides selected for the regression analysis may improve the ability to detect LEU in small samples using the RIID scintillation detectors. This hypothesis requires additional testing and confirmation. The direct scaling of alpha specific activity with ${ }^{235} \mathrm{U}$ enrichment is complicated by the unknown additional enrichment of ${ }^{234} \mathrm{U}$ in the enrichment process.

An additional complication arises from recycled HEU. For example, the 502 sample was determined to contain other isotopes $\left({ }^{233} \mathrm{U},{ }^{241} \mathrm{Am}\right.$ and $\left.{ }^{239} \mathrm{Pu}\right)$ indicating it had been in a reactor and then chemically separated from the fission products. This represents a unique case for uranium enrichment determination with low resolution spectrometers. The spectra of radiations obtained from the RIIDs used in this study were dominated by the ${ }^{241} \mathrm{Am}$ radioisotope present in the spectra due to its high gamma specific activity. This shortcoming was overcome by interposing a thin $(0.6 \mathrm{~cm})$ sheet of steel between the source and the detector prior to accumulating the spectrum. This external absorber effectively reduced the $60 \mathrm{keV}$ gamma ray from ${ }^{241} \mathrm{Am}$ to a level where the $186 \mathrm{keV}$ line was clearly visible in the spectrum and permitted a positive ${ }^{235} \mathrm{U}$ identification. This is a technique suggested for field inspection personnel to use when detecting a ${ }^{241} \mathrm{Am}$ source before dismissing it as an innocent commercial source.

\section{Conclusions}

The detection and characterization of suspect enriched uranium sources can effectively be accomplished using the present RIIDs which contain small scintillators. While the exact enrichment cannot be obtained with the current embedded spectral algorithms, sufficient information can be extracted from the spectra by technical experts using current spectroscopic methods to confirm the presence of threat materials. The most effective off-line methods for spectral analyses include multiple regression techniques and source modeling. In the maritime environment, a significant improvement in the signal to background ratio permits the detection of the weak signals from HEU at reasonable distances by minimally trained individuals. Provided the field instrument can detect the 
presence of an increased radiation field, identify the spectral components to include ${ }^{235} \mathrm{U}$, and transmit the spectra to a knowledgeable individual for confirmation, the interdiction of possible threat materials can be accomplished.

\section{References}

1. R. T. Kouzes, Detecting Illicit Nuclear Materials, American Scientist, Vol. 93, (2005), 422-427.

2. K. J. HOFSTETTER AND D. M. BEALS, Comparison of CdTe and CdZnTe detectors for field determination of uranium isotopic enrichments, J. Radioanalytical and Nuclear Chemistry, Vol. 263, No. 1, (2005) 171-176.

3. Maritime Background Radiation Program, US Coast Guard Research and Development Center, (Department of Homeland Security, Report in process).

4. DEAN J. MITCHELL, HOWARD M. SANGER AND KEITH W. MARLOW, Gamma-Ray Response Functions for Scintillation and Semiconductor Detectors, Nucl. Inst. and Meth. A276 (1989) 547. 


\section{LIST OF FIGURES}

Figure 1. 30-second spectrum of HEU taken with identiFINDER-U

Figure 2. Spectra of a $68 \%$ enriched ${ }^{235} \mathrm{U}$ source taken with each RIID

Figure 3. A spectrum of a Fiestaware plate as analyzed by GADRASw.

Figure 4 . The low energy portion of a $10 \%$ enriched uranium sample

Figure 5. Gamma spectrum with uranium X-rays included.

Figure 6. HPGe spectrum of a $68 \%$ enriched ${ }^{235} \mathrm{U}$ sample without (A) and with (B) the uranium X-ray component.

\section{TABLES}

Table 1. Calculated enrichment by GADRASw with and without inclusion of the X-ray component based on integrated count rates of each component

\begin{tabular}{|c|c|c|c|}
\hline Sample & $\begin{array}{c}\text { True } \\
\text { Isotopic }\end{array}$ & Measured & $\begin{array}{c}\text { Measured with } \\
\text { X-ray Component }\end{array}$ \\
\hline $\mathbf{5 0 4}$ & $\mathbf{1 0 0} \%$ & $\mathbf{8 5 . 5} \%$ & $\mathbf{9 1} \%$ \\
\hline $\mathbf{5 0 2}$ & $\mathbf{9 0} \%$ & $\mathbf{9 0 . 7} \%$ & $\mathbf{9 0 . 9} \%$ \\
\hline SRM & $\mathbf{1 0} \%$ & $\mathbf{9 . 2} \%$ & $\mathbf{1 0 . 7} \%$ \\
\hline V1 & $\mathbf{6 8} \%$ & $\mathbf{4 3} \%$ & $\mathbf{5 9 . 7} \%$ \\
\hline V2 & $\mathbf{0 . 5 3} \%$ & $\mathbf{0 . 6 8} \%$ & $\mathbf{0 . 4 9} \%$ \\
\hline V3 & $\mathbf{0 . 7 1} \%$ & $\mathbf{0 . 8 6} \%$ & $\mathbf{0 . 7 0} \%$ \\
\hline
\end{tabular}


live-time $=29.00$

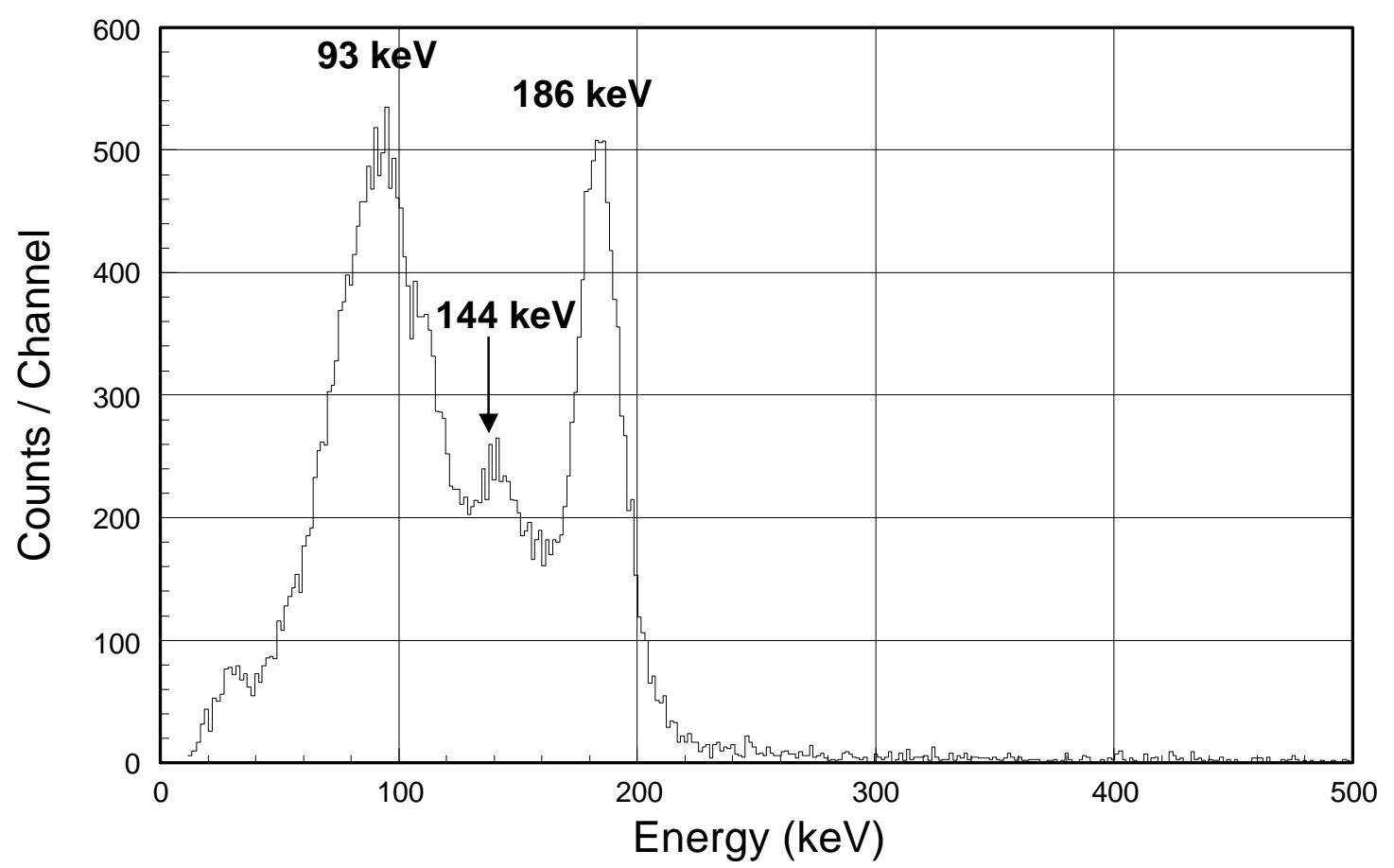

Figure 1. 
IdentiFINDER-U

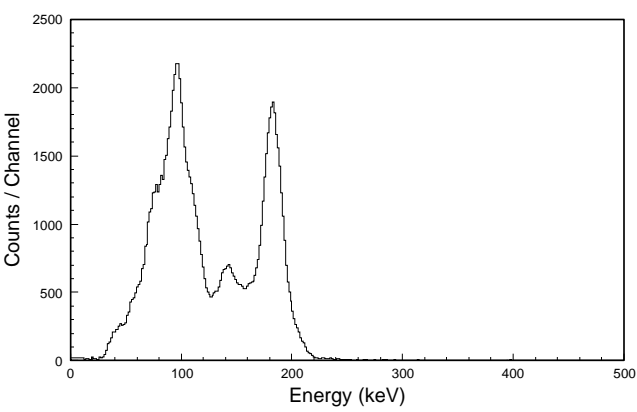

SAM-935

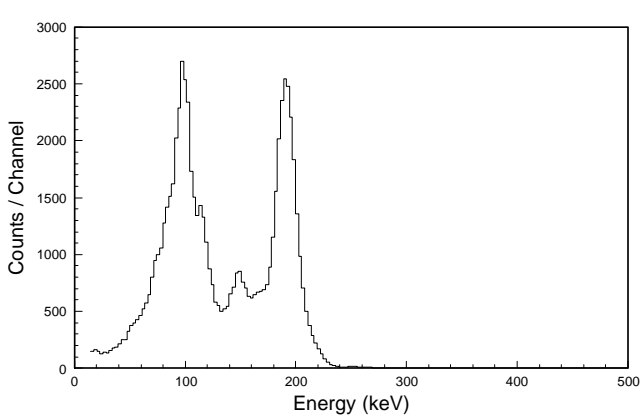

GR-135

live time $=5000$

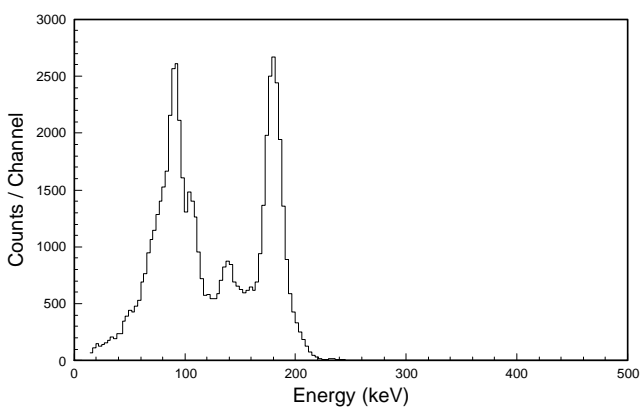

SAM-935 EXT.

live-time $=60.00$

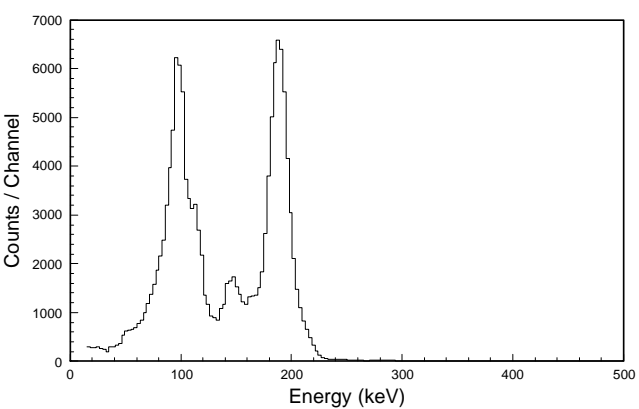

Figure 2. 
Fiestaware

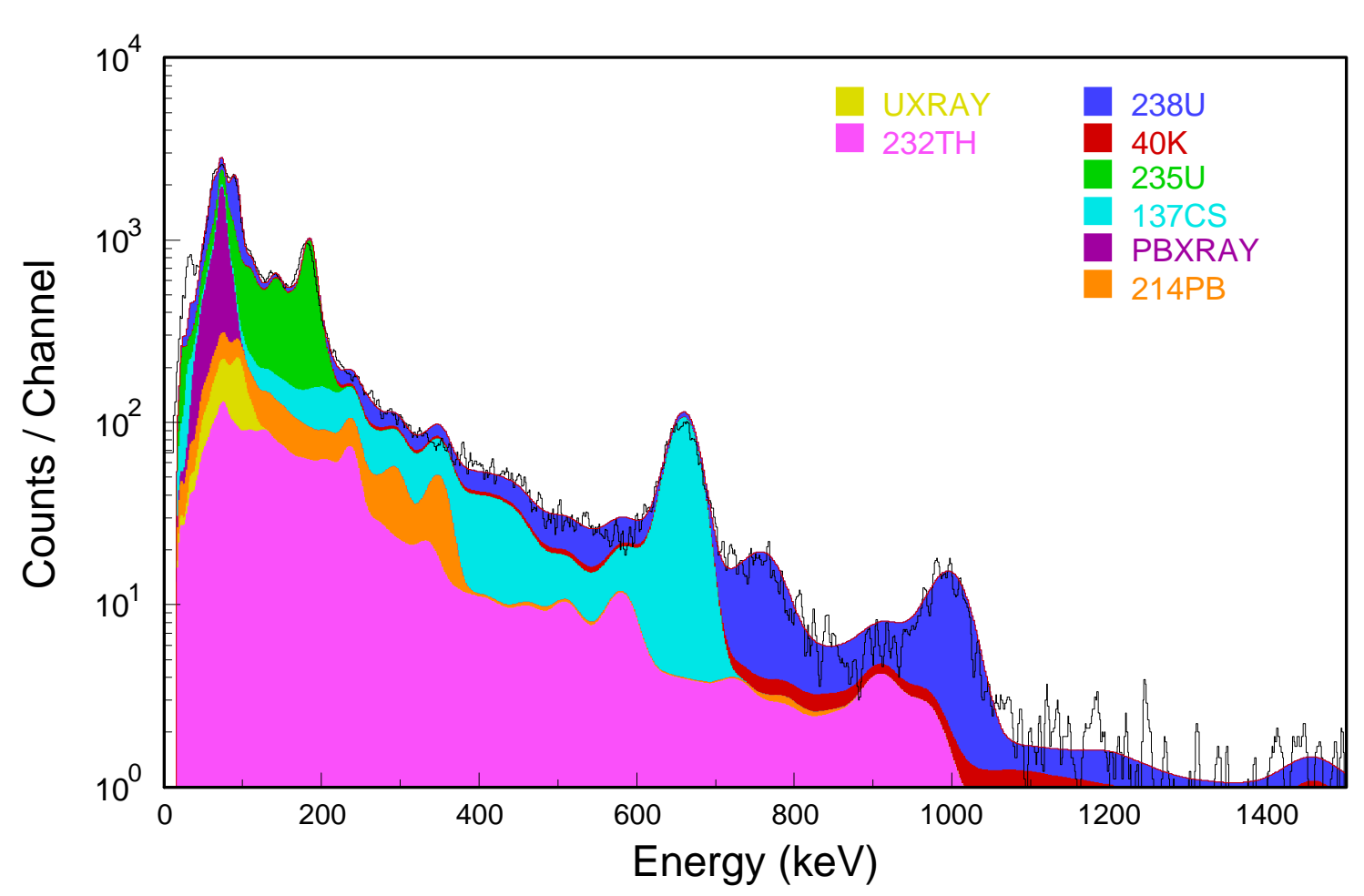

live-time $=172.00$

chi-square $=3.65$

Figure 3. 
NIST@2"

live-time $=59.00$

chi-square $=3.68$

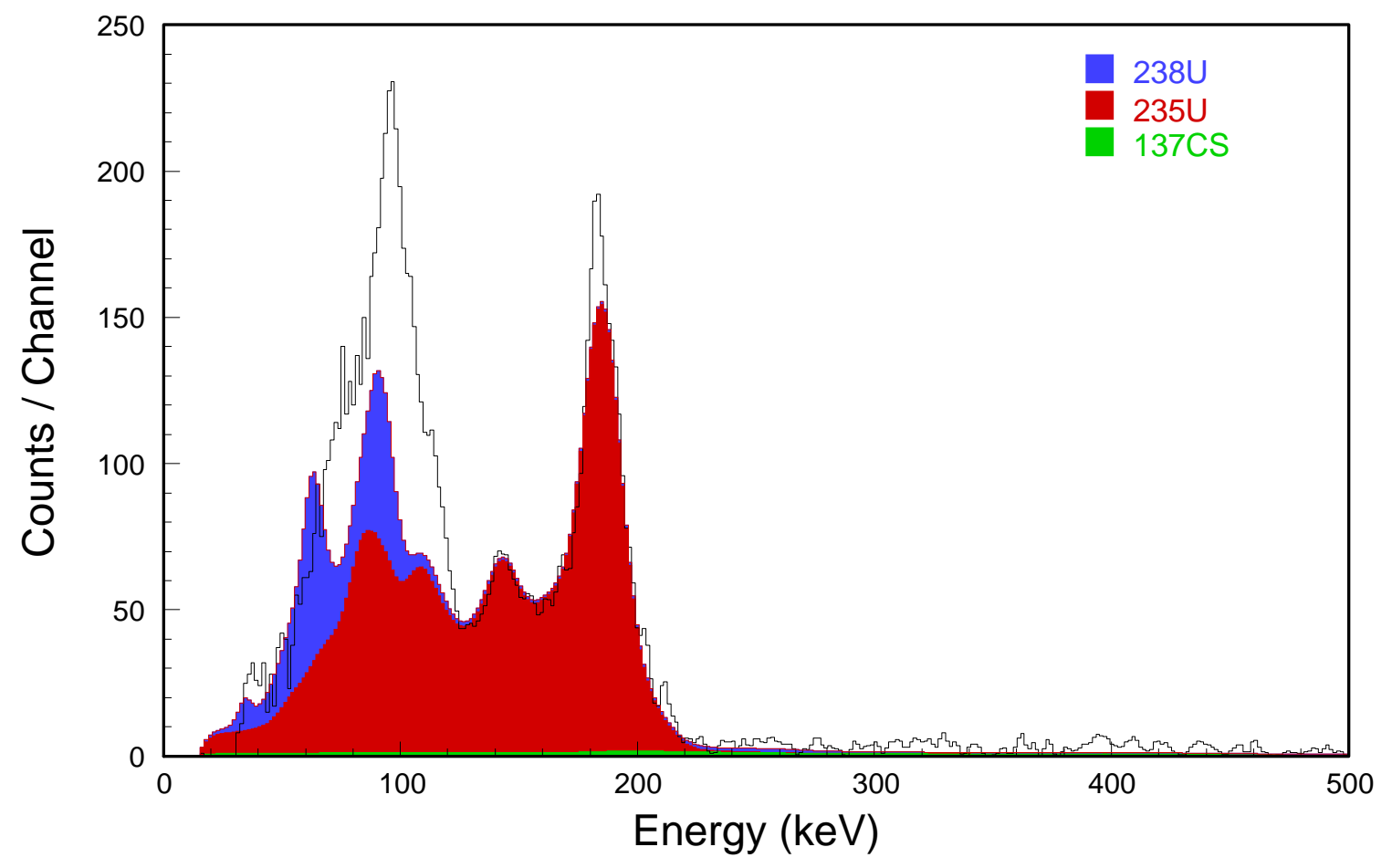

Figure 4. 
NIST@2"

live-time $=59.00$

chi-square $=1.46$

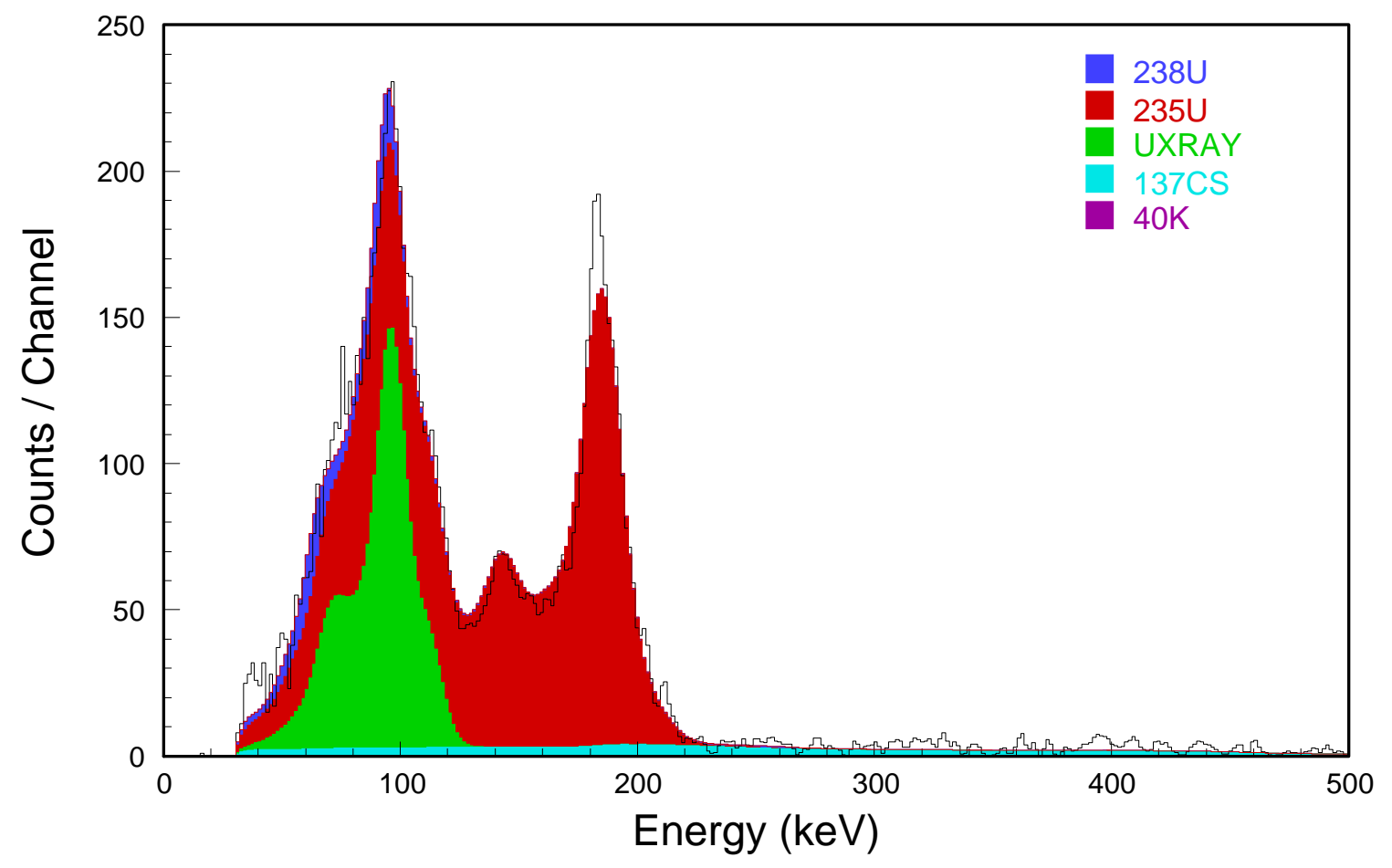

Figure 5. 
live-time $=68.00$

chi-square $=1.60$
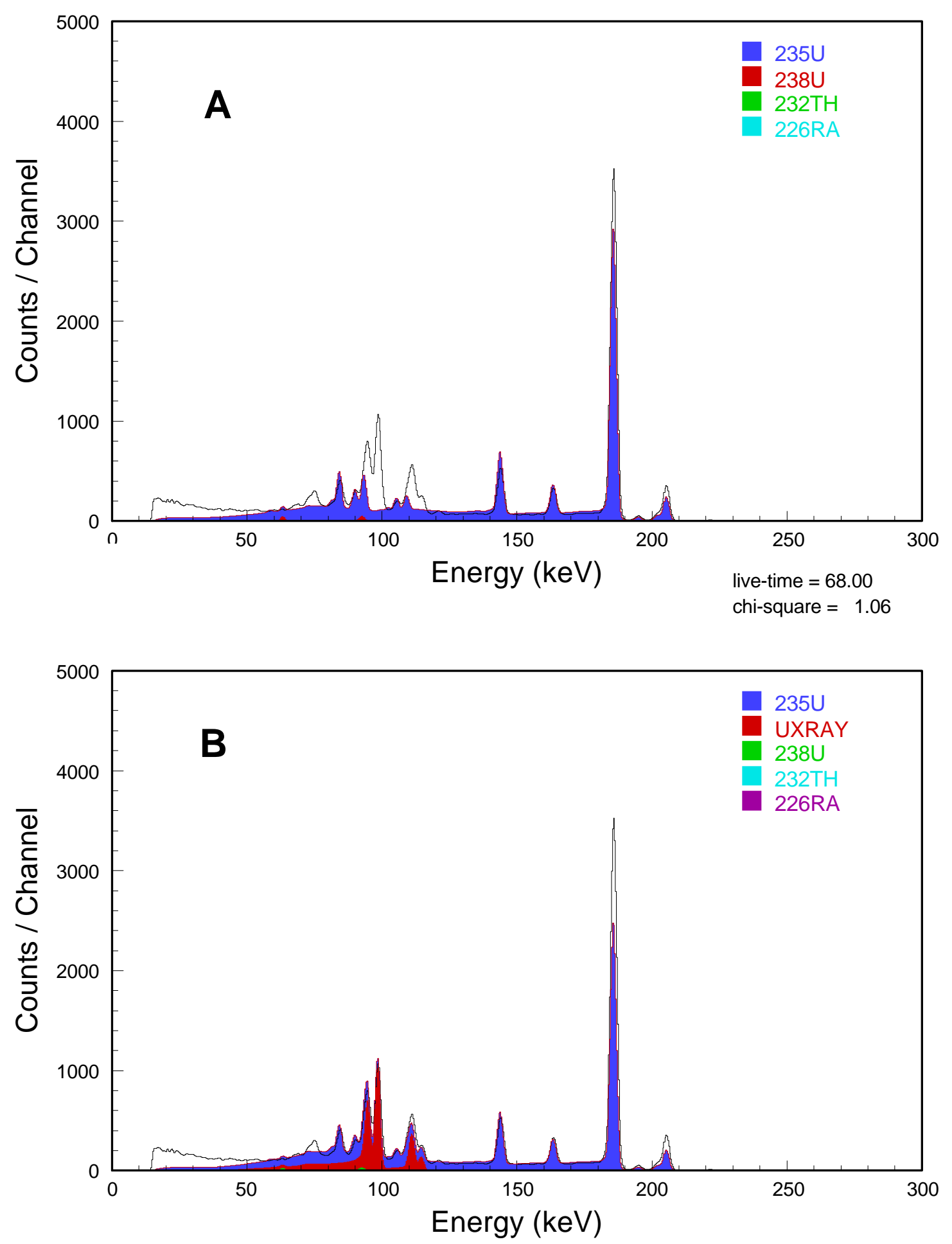